\title{
A Next-to-Minimal model of Hybrid Inflation in Effective Supergravity Theoriest
}

\author{
M. Bastero-Gil and S. F. King \\ Department of Physics and Astronomy, University of Southampton, \\ Southampton, SO17 1BJ, U.K.
}

\begin{abstract}
We propose a model of inflation based on a simple variant of the NMSSM, called $\phi$ NMSSM where the additional singlet $\phi$ plays the role of the inflaton in hybrid (or inverted hybrid) type models. The $\phi$ NMSSM solves the $\mu$ problem of the MSSM via the vacuum expectation value of the gauge singlet $\mathrm{N}$, but also solves the strong CP problem through an approximate Peccei-Quinn symmetry. The potential energy which drives inflation originates from the F-term of the effective supergravity theory which result from a generic string theory. In the class of models considered the inflaton is protected from receiving mass during inflation by a Heisenberg symmetry, avoiding the $\eta$ problem.
\end{abstract}

\section{Introduction}

Due to its intrinsic elegance, inflation [1] has become the almost universally accepted dogma for accounting for the flatness and homogeneity of the universe. One of the most popular versions of inflation these days is hybrid inflation, where there are (at least) two fields at work: the slowly rolling inflaton field $\phi$, and a second field $N$ whose role is to end inflation by developing a non-zero vacuum expectation value (VEV) when $\phi$ passes a certain critical value $\phi_{c}$ during its slow roll. During inflation $N=0$, and the potential along the $\phi$ direction is approximately flat, with the flatness lifted by a $\phi$ mass which must be small enough to satisfy the slow-roll conditions. Given a particular model, the slow roll conditions and COBE constraints on the spectrum of density perturbations then determine the relationship between the height of the potential $V(0)^{1 / 4}$ and the inflaton mass during inflation. However, the origin of the vacuum energy $V(0)$ which drives inflation can only be properly understood within a framework which allows the possibility for the potential energy to settle to zero at the global minimum, and hence lead to an acceptable cosmological constant, and this implies supergravity (SUGRA). In SUGRA models a non-zero vacuum energy can be generated through the F-terms and/or the D-terms, but here we will assume the D-terms to be negligible or zero.

In F-term Hybrid inflation we have to face the so-called $\eta$ problem. During inflation SUSY is broken by non-zero F-terms, and due to the exponential factor for the Kahler potential in front of the potential all the scalar fields including the inflaton will pick up masses of the order of the Hubble constant, $H \approx V(0)^{1 / 2} / \tilde{M}_{P}$.

\footnotetext{
${ }^{1}$ Talk given at International Workshop on Particle Physics and the Early Universe, Monterey, California, 15-20 Nov 1998.
} 
This will lead to a violation of the slow roll condition $|\eta|=\tilde{M}_{P}^{2}\left|V^{\prime \prime} / V\right|^{2} \ll 1$. To overcome this problem different solutions can be found in the literature [2]. Here we would like to pursue the possibility that the inflaton mass remains zero at tree-level during inflation and its only contribution is given by very small radiative corrections, safely smaller than the Hubble constant. This can be achieved working in the context of no-scale SUGRA theories, where it is known that the soft scalar masses can be zero even in the presence of a non-zero gravitino mass. Moreover, the requirement of small inflaton mass, combined with the COBE constraint, imply that the height of the potential during inflation must be lower than the usual SUSY breaking scale, $M_{S U S Y} \approx 10^{11} \mathrm{GeV}$, and we will show how the no-scale structure allows this possibility [3]. We shall give an explicit example where the SUSY breaking sector, the height of the potential and the inflaton sector are all specified.

\section{The model}

The model is based on the superpotential [4:

$$
\tilde{W}=\lambda N H_{1} H_{2}-k \phi N^{2}
$$

with the fields $\phi, N$ being gauge singlets, and $H_{1}, H_{2}$ the minimal supersymmetric standard model Higgs doublets. Since the VEV of $N$ generates the effective $\mu$ mass term coupling the two Higgs doublets, we require that $\lambda\langle N\rangle \sim 1 \mathrm{TeV}$ as in the well known particle physics next-to-minimal supersymmetric standard model (NMSSM) [5]. This superpotential is invariant under a global $U(1)_{P Q}$ symmetry, broken by the VEVs of $\phi$ and $N$, and which leads to a very light axion with its decay constant of order of the VEVs $<N>\sim<\phi>$. This implies $<N>\sim 10^{13} \mathrm{GeV}$, in order to satisfy the cosmological axion bounds. The Higgs doublets develop electroweak VEVs, much smaller than $\langle N\rangle$, and they may be ignored in the analysis.

The potential relevant for inflation then reads,

$$
V(\phi, N)=V(0)+k^{2} N^{4}+\left(m_{N}^{2}-2 k A_{k} \phi+4 k^{2} \phi^{2}\right) N^{2}+m_{\phi}^{2} \phi^{2}
$$

where the soft parameters above occur in the soft SUSY breaking potential, and they are typically of the order of $1 \mathrm{TeV}$, but $\mathrm{m}_{\phi}^{2}$ owes its origin to radiative corrections to the potential controlled by the small coupling $k$. We have added a constant vacuum energy $V(0)$ to the potential, whose origin we explain latter.

For large values of the field $\phi$ the effective $N$ mass is positive and during inflation the field $N=0 ; \phi$ slowly rolls until it reaches a critical valuef $\phi_{c} \approx \frac{A_{k}}{4 k}$. When the critical value is reached, inflation ends and the global minimum is achieved, with non zero VEVS $<\phi>\sim<N>\sim \phi_{c}$. After inflation ends $V(0)$ is assumed to remain unchanged, but be cancelled by a negative contribution from the remaining part of the potential at the global minimum, $\left.V(<\phi>,<N>)=-k^{2}<N\right\rangle^{4}$. Due to the axion bound, we then have $k \approx 10^{-10}$, and $V(0)^{1 / 4} \approx 10^{8} \mathrm{GeV}$. To satisfy the COBE

${ }^{2}$ In fact the model has two different critical values, $\phi_{c}^{ \pm}$, which allows the possibility of having either standard hybrid inflation or inverted hybrid inflation [6] depending on the sign of the mass squared $m^{2}$. 
constraint the model requires an inflaton mass in the range of a few $\mathrm{eV}$, and this is consistent with this mass being generated by radiative corrections controlled by the small coupling] $k$.

We now wish to elevate this model to an effective no-scale SUGRA theory, where the tree-level inflaton mass is ensured to vanish. This kind of theory may be obtained from $4 \mathrm{~d}$ effective string theories [7]. We place the inflaton and the $N$ field in the untwisted sector (modular weight -1) along with the moduli fields, and assume the following conditions to hold during inflation:

(a) The superpotential is independent of the over-all modulus $T$ and, together with Eq. (11), it includes a dilaton superpotential $W(S)=\Lambda^{3} e^{-S / b_{0}}$. The dilaton will act as a source for SUSY breaking, with the gravitino mass given by:

$$
m_{3 / 2}^{2} \simeq e^{K} \frac{|W(S)|^{2}}{\tilde{M}_{P}^{4}} \simeq \frac{\Lambda^{6}}{\tilde{M}_{P}^{4}}
$$

The requirement of having a gravitino mass $m_{3 / 2} \approx 1 \mathrm{TeV}$ then fixes the effective scale $\Lambda$ to be of the order of $10^{13} \mathrm{GeV}$.

(b) The Kahler potential is given by,

$$
K=-3 \ln (\rho)-\ln \left(S+S^{*}\right)+\frac{\beta}{\rho^{3}}-\frac{2 s_{0}}{S+S^{*}}+\frac{b+4 s_{0}^{2}}{6\left(S+S^{*}\right)^{2}},
$$

It depends only on the combination $\rho=T+T^{*}-\sum_{i} \phi_{i} \phi_{i}^{*}$, with $\phi_{i}$ any untwisted field of the theory, in particular $\phi$ and $N$. This condition can be formalised in terms of a Heisenberg symmetry [8]. The twisted fields are switched off during inflation, and they do not contribute. We remark that we only demand the theory to posses a Heisenberg symmetry during inflation. After inflation ends this symmetry may or may not be broken by the contribution from the twisted sector. The last three terms of Eq. (舟) model non-perturbative terms for both the field $\rho$ and the dilaton $S$ [9], needed in order to stabilise them at a fixed value during inflation, $\rho_{0} \approx(2 \beta)^{1 / 3}$ and $2 R e S_{0} \approx s_{0}$.

The above conditions ensure that the inflaton remains massless at tree-level. The key point is that $\rho$ is fixed at its minimum, for which the condition $d V / d \rho=0$ is fulfilled. Then, computing the mass matrix for the fields $(T, \phi)$ during inflation it can be shown that there is a zero eigenvalue which corresponds to the massless inflaton.

The non-zero potential $V(0)$ is given by the SUGRA potential:

$$
V=\left|F_{T}\right|^{2}+\left|F_{S}\right|^{2}-3 m_{3 / 2}^{2} \tilde{M}_{P}^{2}
$$

evaluated at the minimum $\rho_{0}$ and $S_{0}$. If we now require that the potential vanish in the global minimum at the end of inflation, when the fields $\phi$ and $N$ also contribute, then we obtain that during inflation the height of the potential is $V^{1 / 4} \sim \epsilon^{1 / 4} \sqrt{m_{3 / 2} \tilde{M}_{P}}$,

\footnotetext{
${ }^{3}$ The smallness of $k$ seems to indicate that it has a non-renormalisable origin; this may involve an additional sector which obeys a discrete $Z_{3} \times Z_{5}$ symmetry from which the Peccei-Quinn symmetry emerges as an approximation (for details see [4]).
} 
with $\epsilon^{1 / 4} \sim 10^{-3}$. In our approach, the fact that the potential is much smaller than the typical SUSY breaking scale is a consequence of having a very small coupling $k$, otherwise needed to control the radiative corrections to the inflaton mass. Another good feature of such a small coupling is that the contribution of the hybrid superpotential at the end of inflation will be highly suppressed with respect to the dilaton contribution, and then the minima for the moduli and the dilaton (and the gravitino mass) are mainly the same during and after inflation, i. e., no cosmological moduli problem is present in our scenario. As discussed in [G] the small couplings $\lambda, k$ may have a natural explanation in terms of non-renormalisable effective operators.

\section{References}

[1] See for example Lyth, D. H., and Riotto, A., hep-ph/9807278, and references therein.

[2] See for example Covi, L., in this proceedings.

[3] Bastero-Gil, M., and King, S. F., hep-ph/9806477.

[4] Bastero-Gil, M., and King, S. F., Phys. Lett. B423, 27 (1998).

[5] Fayet, P., Nucl. Phys. B90, 104 (1975); Nilles, H. -P., Srednicki, M., and Wyler, D., Phys. Lett. B120, 346(1983); Derendinger, J. -P., and Savoy, C. A., Nucl. Phys. B237, 307(1984); Ellis, J., et al., Phys. Rev. D39, 844 (1989); Durand, L., and Lopez, J. L., Phys. Lett. B217, 463 (1989); Drees, M., Int. J. Mod. Phys. A4, 3635 (1989); King, S. F., and White, P. L., Phys. Rev. D52, 4183 (1995).

[6] Lyth, D. H., and Stewart, E. D., Phys. Rev. D54, 7186 (1996); King, S. F., and Sanderson, J., Phys. Lett. B412, 19 (1997).

[7] Ferrara, S., Kounnas, C., and Porrati, M., Phys. Lett. B181, 263 (1986); Cvetic, M., Louis, J., and Ovrut, B., ibid B206,227 (1988); Ibáñez, L. E., and Lüst, D., Nucl. Phys. B382,305 (1992).

[8] Binetruy, P., and Gaillard, M. K., Phys. Lett. B195, 382 (1987); Gaillard, M. K., Murayama, H., and Olive, K., ibid B355, 71 (1995); Gaillard, M. K., Lyth, D. H., and Murayama, H., Phys. Rev. D58, 1235 (1998).

[9] Banks, T., and Dine, M., Phys. Rev. D50, 7454 (1994); Casas, J. A., Phys. Lett. B384, 103 (1996); Binétruy, P., Gaillard, M. K. and Wu, Y.-Y, Nucl. Phys. B481, 109 (1996); Barreiro, T., de Carlos, B. and Copeland, E. J., Phys. Rev. D57, 7354 (1998); for the dilaton non-perturbative Kahler potential we follow the particular ansatz given in: Arkani-Hamed, N., Dine, M., and Martin, S., Phys. Lett. B431, 329 (1998). 